\title{
Die PASSION-Studie
}

\section{Randomized comparison of PAclitaxel eluting Stent versus conventional stent in ST-elevation myocardial infarctION}

Hintergrund und Problemstellung: Medikamente freisetzende Stents (DES) reduzieren die Notwendigkeit einer erneuten Revaskularisation nach PCI bei Patienten mit stabiler KHK. In Registern gibt es Hinweise, dass DES auch bei Patienten mit ST-Strecken-Hebungsinfarkt (STEMI) die Prognose ver-

\begin{tabular}{ll}
\hline Methodik & \\
\hline Studiendesign & Randomisiert, kontrolliert \\
Primärer Endpunkt & $\begin{array}{l}\text { MACE nach 1 Jahr, (definiert als ischämieindi- } \\
\text { zierte TLR durch Re-PCI oder Bypass-OP des } \\
\text { Zielgefäßes, Reinfarkt, Herztod) }\end{array}$ \\
Patientenauswahl & $\begin{array}{l}\text { Einschlusskriterien: } \\
\text { Patienten mit STEMI }<12 \mathrm{~h} \text {, die eine primäre } \\
\text { PCI benötigen } \\
\text { ST-Hebung } \geq 2 \mathrm{~mm} \text { in } \geq 2 \text { Ableitungen } \\
\text { De-novo- Stenose } \\
\text { Ausschlusskriterien: } \\
\text { erfolglose Fibrinolyse } \\
\text { kardiogener Schock } \\
\text { künstliche Beatmung }\end{array}$ \\
& 2 \\
\hline $\begin{array}{l}\text { Anzahl der teil- } \\
\text { nehmenden Zentren }\end{array}$ & \\
\hline
\end{tabular}

\begin{tabular}{|c|c|c|}
\hline \multicolumn{3}{|l|}{ Ergebnisse $\left({ }^{*} p<0,05\right)$} \\
\hline & $\begin{array}{l}\text { Kontrollgruppe } \\
\text { (Express, Liberté) }\end{array}$ & Taxus \\
\hline Patientenzahl & $\mathrm{n}=310$ & $\mathrm{n}=309$ \\
\hline $\begin{array}{l}\text { Ausgangsdaten: } \\
\text { Patienten- } \\
\text { charakteristika }\end{array}$ & \multicolumn{2}{|c|}{$\begin{array}{l}\text { Beide Gruppen waren hinsichtlich der Standardaus- } \\
\text { gangsparameter vergleichbar } \\
\text { Stentgröße: BMS/DES: } 3,3 / 3,2 \mathrm{~mm} \\
\text { Stentlänge: BMS/DES: } 19 / 19 \mathrm{~mm} \\
\text { Anzahl der Stents: BMS/DES: 1,3/1,3 }\end{array}$} \\
\hline $\begin{array}{l}\text { Primärer Endpunkt } \\
\text { (s. o.) }\end{array}$ & $12,6 \%$ & $8,7 \%$ \\
\hline \multicolumn{3}{|l|}{$\begin{array}{l}\text { Klinische Parameter } \\
\text { (12 Monate): }\end{array}$} \\
\hline Tod oder Myokardinfarkt & $6,5 \%$ & $4,8 \%$ \\
\hline Gesamte Stentthrombosen & $1 \%$ & $1 \%$ \\
\hline $\begin{array}{l}\text { TLR (target lesion } \\
\text { revascularization) }\end{array}$ & $7,4 \%$ & $6,2 \%$ \\
\hline
\end{tabular}

Herz 2006;31:170

DOI 10.1007/s00059-006-2812-z bessern. Ziel der Studie war der Nachweis von Wirksamkeit und Sicherheit des Taxus-Stents im Vergleich zu unbeschichteten Stents (BMS) bei Patienten mit STEMI. Studienleiter war M. Dirksen, Amsterdam, Niederlande.

\begin{tabular}{|c|c|c|}
\hline \multicolumn{3}{|c|}{ Evidence-based-Medicine (EBM-) Score (www.TCTMD.com) } \\
\hline $\begin{array}{l}\text { Klinischer primärer } \\
\text { Endpunkt }\end{array}$ & $\mathrm{Ja}=3$, nein $=0$ & 3 \\
\hline $\begin{array}{l}\text { Doppelblind } \\
\text { (einschließlich Ärzte) }\end{array}$ & $\mathrm{Ja}=1$, nein $=0$ & 0 \\
\hline $\begin{array}{l}\text { Beobachtungsintervall } \\
\text { für den primären } \\
\text { Endpunkt } \geq 6 \text { Monate }\end{array}$ & $\mathrm{Ja}=1$, nein $=0$ & 1 \\
\hline $\begin{array}{l}\text { Multicenter } \\
\text { (mindestens } 3 \text { Zentren) }\end{array}$ & $\mathrm{Ja}=1$, nein $=0$ & 0 \\
\hline $\begin{array}{l}\text { Externes und vom } \\
\text { Steering Committee unab- } \\
\text { hängiges Clinical Event } \\
\text { Committee / DSMB } \\
\text { (Datensicherheit } \\
\text { Monitoring Board) }\end{array}$ & $\mathrm{Ja}=1$, nein $=0$ & $(?)$ \\
\hline Primärer Endpunkt erreicht & $\mathrm{Ja}=1$, nein $=0$ & 0 \\
\hline $\begin{array}{l}\text { Power von } \geq 80 \% \text { für den } \\
\text { primären Endpunkt erreicht }\end{array}$ & $\mathrm{Ja}=1$, nein $=0$ & 0 \\
\hline $\begin{array}{l}\text { Anteil des Follow-up der Patienten } \\
\text { für angiographischen primären } \\
\text { Endpunkt } \geq 80 \% \text { und } \geq 95 \% \text { für } \\
\text { klinischen primären Endpunkt }\end{array}$ & $\mathrm{Ja}=1$, nein $=0$ & 1 \\
\hline \multicolumn{2}{|c|}{$\begin{array}{l}\text { Gesamt-EBM-Score } \\
\text { („Silber-Score“ für randomisierte Studien) }\end{array}$} & 5 \\
\hline
\end{tabular}

Schlussfolgerung und Kommentar: PASSION ist die erste randomisierte Studie, die anhand eines primären klinischen Endpunkts den Taxus-Stent mit BMS bei STEMI-Patienten verglich. Der primäre Endpunkt (MACE nach 1 Jahr) wurde nicht erreicht. Da eine routinemäßige Kontrollangiographie nicht im Protokoll vorgesehen war, kann zum Late Loss keine Aussage gemacht werden. Der Vergleich zu TYPHOON (s. dort) stellt die Frage nach eventuellen Unterschieden der beiden DES - aber auch eventuelle Qualitätsunterschiede der jeweils in den Kontrollgruppen verwendeten BMS mögen eine Rolle spielen. So stellt sich die Frage, weshalb die 1-JahresMACE-Rate in der TYPHOON-Kontrollgruppe mit $14,6 \%$ höher lag als in der PASSION-Kontrollgruppe mit 12,6\%. Auch wurden in PASSION Patienten mit Bifurkationsstenosen und hoher Thrombuslast eingeschlossen, die in TYPHOON ausgeschlossen waren. Entscheidender Unterschied zwischen TYPHOON und PASSION ist die geplante bzw. bzw. nicht vorgesehene Kontrollangiographie („okulostenotischer Reflex"?). Letztendlich können die offenen Fragen nur durch eine „Head-to-Head“-Studie (Cypher vs. Taxus bei Patienten mit STEMI) geklärt werden. 